\title{
Electrical Stimulation to Enhance Spinal Fusion: A Systematic Review
}

\author{
Paul Park ${ }^{1}$ Darryl Lau ${ }^{2}$ Erika D. Brodt ${ }^{3}$ Joseph R. Dettori ${ }^{3}$ \\ ${ }^{1}$ Department of Neurological Surgery, University of Michigan Health \\ System, Ann Arbor, Michigan, United States \\ 2 Department of Neurological Surgery, University of California San \\ Francisco, San Francisco, California, United States \\ Address for correspondence Paul Park, MD, Department of \\ Neurological Surgery, University of Michigan Health System, 3552 \\ Taubman Center, Box 0338, 1500 East Medical Center Drive, Ann \\ Arbor, MI 48109, United States (e-mail: ppark@med.umich.edu).
}

${ }^{3}$ Spectrum Research, Inc., Tacoma, Washington, United States

Evid Based Spine Care J 2014;5:87-94.

\begin{abstract}
Study Design Systematic review.

Clinical Questions Compared with no stimulation, does electrical stimulation promote bone fusion after lumbar spinal fusion procedures? Does the effect differ based on the type of electrical stimulation used?

Methods Electronic databases and reference lists of key articles were searched up to October 15, 2013, to identify randomized controlled trials (RCTs) comparing the effect of electrical stimulation to no electrical stimulation on fusion rates after lumbar spinal fusion for the treatment of degenerative disease. Two independent reviewers assessed the strength of evidence using the Grades of Recommendation Assessment, Development and Evaluation (GRADE) criteria.

Results Six RCTs met the inclusion criteria. The following types of electrical stimulation were investigated: direct current (three studies), pulsed electromagnetic field (three studies), and capacitive coupling (one study). The control groups consisted of no stimulation (two studies) or placebo (four studies). Marked heterogeneity in study populations, characteristics, and design prevented a meta-analysis. Regardless of the type of electrical stimulation used, cumulative incidences of fusion varied widely across

Keywords

- electrical stimulation

- direct current

- pulsed electromagnetic field

- capacitive coupling

- fusion

- lumbar spine the RCTs, ranging from 35.4 to $90.6 \%$ in the intervention groups and from 33.3 to $81.9 \%$ in the control groups across 9 to 24 months of follow-up. Similarly, when stratified by the type of electrical stimulation used, fusion outcomes from individual studies varied, leading to inconsistent and conflicting results.

Conclusion Given the inconsistency in study results, possibly due to heterogeneity in study populations/characteristics and quality, we are unable to conclude that electrical stimulation results in better fusion outcomes compared with no stimulation. The overall strength of evidence for the conclusions is low.
\end{abstract}

\section{Study Rationale and Context}

Degenerative spinal conditions can lead to pain and neurologic symptoms. Patients who do not respond to nonoperative treatment often undergo spinal fusion. The lack of significant bone formation resulting in nonunion of the treated spinal segments, known as a pseudoarthrosis, is a potential longterm complication of a spinal fusion procedure. Although application of rigid instrumentation, such as the pedicle screw-rod construct, has increased fusion rates, received

February 28, 2014

accepted after revision

May 12, 2014 (c) 2014 Georg Thieme Verlag KG Stuttgart · New York
DOI http://dx.doi.org/ 10.1055/s-0034-1386752. ISSN 1663-7976. 
pseudoarthrosis still occurs and has been shown to be the cause of persistent or recurrent pain and disability. ${ }^{1,2}$ Revision surgery is often recommended for these symptomatic cases of nonfusion and incidence is not insignificant as pseudoarthrosis is one of the most common indications for repeat surgery. ${ }^{3}$ Consequently, other measures including the use of biologics such as bone morphogenetic proteins or mesenchymal stem cell enriched allograft has been used to further increase the rate of bony union. Electrical stimulation has been suggested as an alternative means for increasing the fusion rate. However, the mechanism and efficacy of electrical stimulation remain unclear. The purpose of this systematic review is to evaluate the various types of stimulation and determine whether electrical stimulation induces bone fusion.

\section{Clinical Question}

Compared with no stimulation, does electrical stimulation promote bone fusion after lumbar spinal fusion procedures? Does the effect differ based on the type of electrical stimulation used (direct current [DC], pulsed electromagnetic field [PEMF], capacitive coupling [CC])?

\section{Materials and Methods}

Study design: Systematic review.

Search: The databases included PubMed, Cochrane collaboration database, and National Guideline Clearinghouse databases; bibliographies of key articles.

Dates searched: The data were searched from January 1980 to October 15, 2013.

Inclusion criteria: (1) Adults, (2) degenerative disease of the lumbar spine, (3) lumbar spinal fusion (any type/approach) with or without instrumentation, (4) comparison of electrical stimulation (including DC, PEMF, and CC) as an adjunctive treatment versus no stimulation, (5) randomized controlled trials (RCTs) published in English in peer-reviewed journals.

Exclusion criteria: (1) Pediatric patients, (2) cancer, trauma, inflammatory arthritis, or osteoporosis as indication for fusion procedure, (3) treatment of the cervical or thoracic spine, (4) use of biologics, (5) animal studies, (6) noncomparative studies (i.e., case series, case reports).

Outcomes: Proportion of patients achieving bony fusion. Analysis: Descriptive statistics. Due to heterogeneity in study populations (including differences in the use of a placebo device, method of fusion assessment, definition of fusion, follow-up length, treatment indications, patient demographics, fusion procedure type/approach, and fusion graft materials), a meta-analysis was not performed.

Overall strength of evidence: Risk of bias for individual studies was based on using criteria set by The Journal of Bone and Joint Surgery ${ }^{4}$ modified to delineate criteria associated with methodological quality and risk of bias based on recommendation from the Agency for Healthcare Research and Quality. ${ }^{5,6}$ The overall strength of evidence across studies was based on precepts outlined by the
Grades of Recommendation Assessment, Development and Evaluation (GRADE) Working Group ${ }^{7}$ and recommendations made by the Agency for Healthcare Research and Quality (AHRQ). ${ }^{5,6}$

Details about methods can be found in the online supplementary material.

\section{Results}

- We identified six RCTs, all rated level of evidence (LoE) II, which met the inclusion criteria and form the basis for this report (-Fig. 1). Further details on the LoE rating for these studies as well as a list of excluded studies can be found in the online supplementary material.

- Three studies compared DC stimulation to no stimulation, ${ }^{8-10}$ with only one study using a placebo device (i.e., an inactive stimulator) in the control group; three compared PEMF stimulation to no stimulation, ${ }^{9,11,12}$ with two employing placebo devices, and one study compared CC to no stimulation using placebo devices. ${ }^{13}$ Marked heterogeneity was present across the studies (-Table 1).

\section{Fusion: Any Electrical Stimulation}

- Regardless of the type of electrical stimulation used, the proportion of patients achieving bony fusion across all six RCTs varied, ranging from 35.4 to $90.6 \%$ compared with 33.3 to $81.9 \%$ in the control groups across 9 to 24 months of follow-up ${ }^{8-13}$ (-Fig. 2).

\section{Fusion: Type of Electrical Stimulation}

\section{Direct Current Stimulation}

- DC stimulation resulted in varying cumulative incidences of fusion ranging from 35.4 to $80.6 \%$ compared with 33.3 to $81.0 \%$ in the control groups across three RCTs with a range of 12 to 24 months follow-up ${ }^{8-10}$ (- Fig. 2).

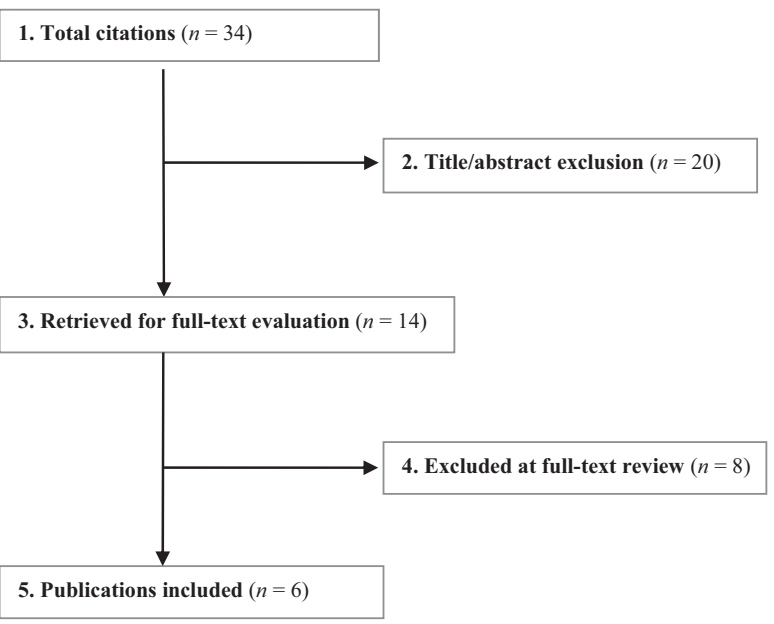

Fig. 1 Flow chart showing results of literature search. 


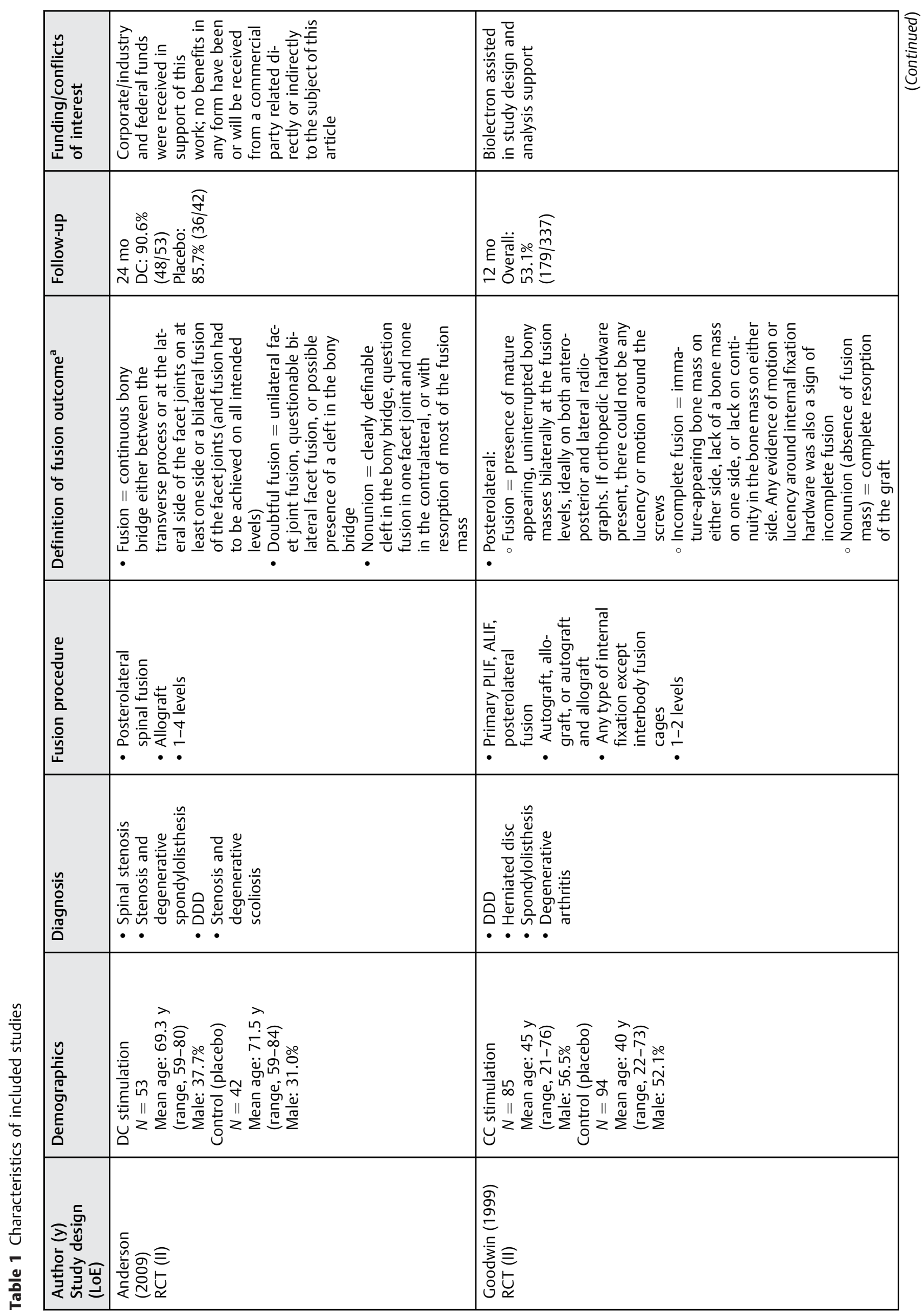




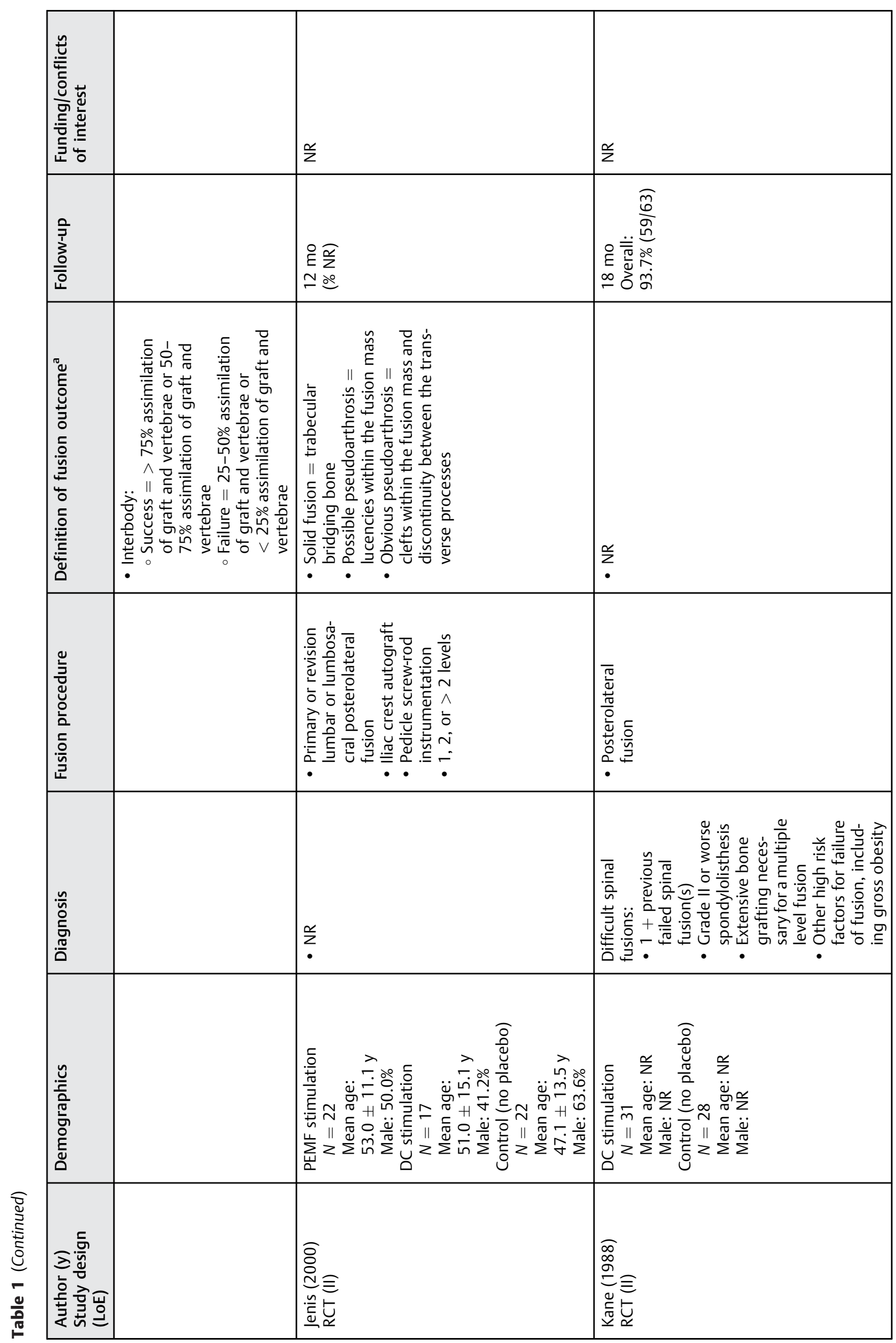




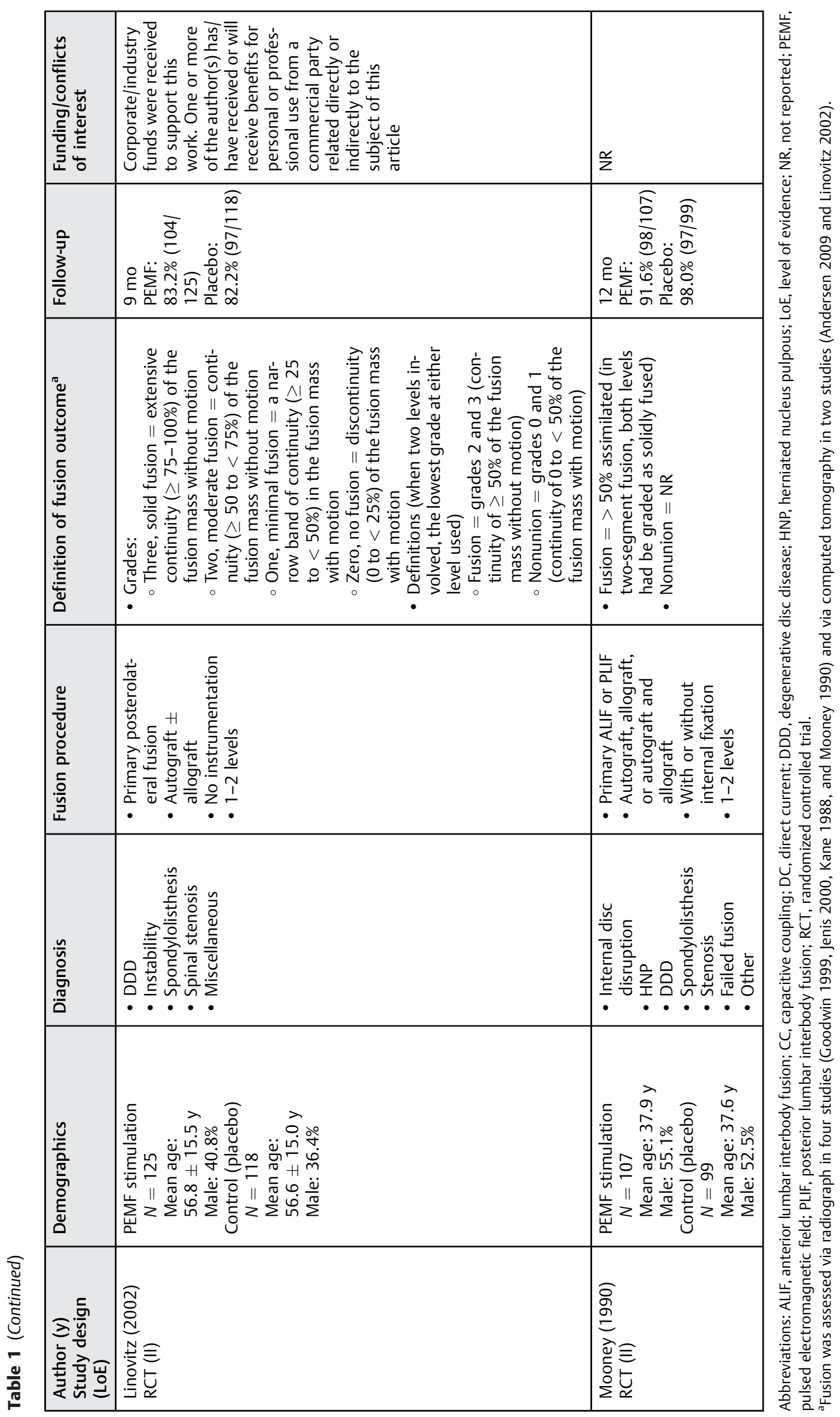




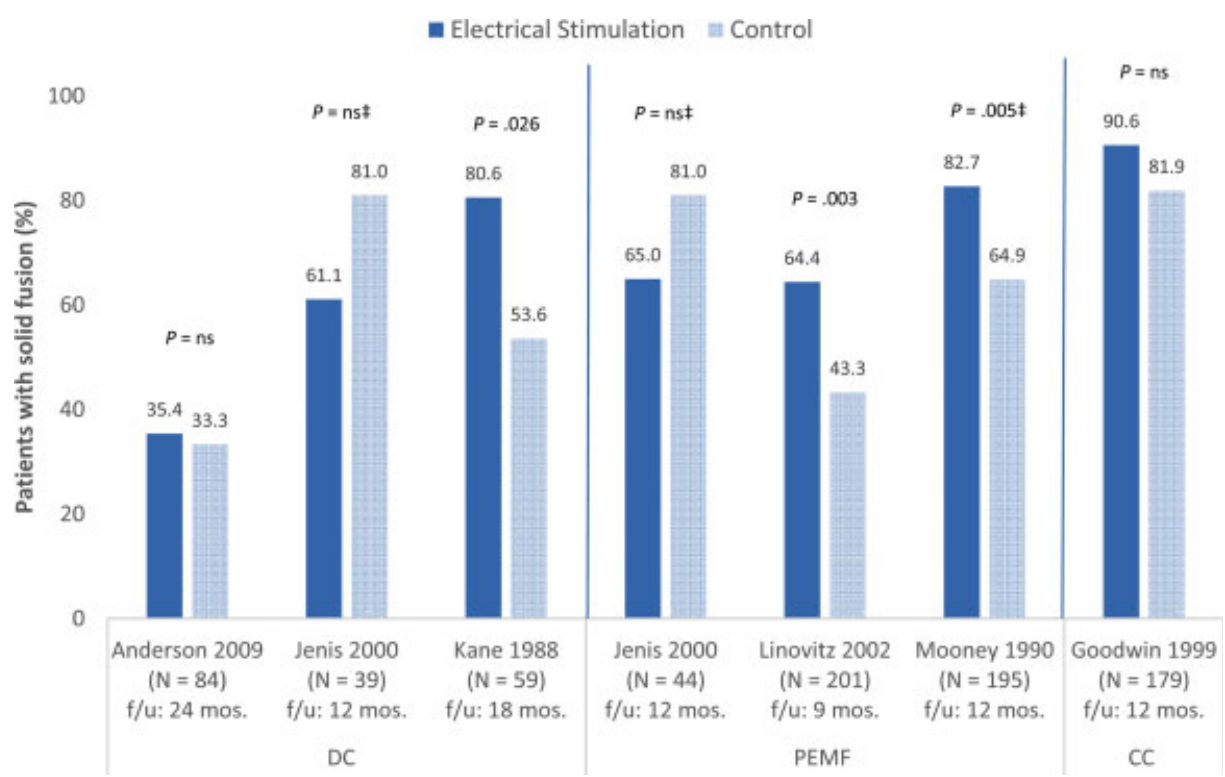

Fig. 2 Proportion of patients that achieved solid fusion in the intervention (electrical stimulation) and the control (placebo/no stimulation)* groups following lumbar spinal fusion procedures ${ }^{\dagger}$. CC, capacitive coupling; DC, direct current; PEMF, pulsed electromagnetic field. ${ }^{*}$ The following RCTs used a placebo device in the control group: Anderson 2009, Goodwin 1999, Linovitz 2002, and Mooney 1990. ${ }^{\dagger}$ Marked heterogeneity in study population and design was present across the six RCTs. Anderson 2009: Elderly population (mean age 70 years); industry sponsored/funded; random sequence generation not reported; no intention-to-treat analysis; did not control for possible confounding factors. Goodwin 1999: Industry sponsored/funded; random sequence generation and statement of concealed allocation not reported; no intention-to treat analysis; unclear if cointerventions were applied equally; $<80 \%$ of patients followed. Jenis 2000: patient diagnoses not reported; funding/conflicts of interest not reported; statement of concealed allocation not reported; no intention-to-treat analysis; < $80 \%$ of patients followed; did not control for possible confounding factors. Kane 1988: Age and \% male not reported; difficult spinal fusions; no definition of fusion outcome provided; funding/conflicts of interest not reported; statement of concealed allocation not reported; no intention-to-treat analysis; unclear if cointervention were applied equally. Linovitz 2002: Industry sponsored/funded; statement of concealed allocation not reported. Mooney 1990: Younger population (mean age 38 years); random sequence generation and statement of concealed allocation not reported; no intention-to-treat analysis; unclear if cointervention were applied equally. ${ }^{\ddagger}$ These studies did not report $\mathrm{p}$ values; $\mathrm{p}$ values were calculated by this article’s authors using the STATA software program.

\section{Pulsed Electromagnetic Field Stimulation}

- Cumulative incidences of fusion following PEMF stimulation compared with no stimulation varied across three RCTS, ranging from 64.4 to $82.7 \%$ and from 43.3 to $81.0 \%$, respectively, over follow-up periods ranging from 9 to 12 months s $^{911,12}$ (-Fig. 2).

\section{Capacitive Coupling Stimulation}

- Only one RCT looked at CC stimulation and reported a similar proportion of patients achieving fusion at 12 months between the intervention and control group: 90.6 versus $81.9 \%$, respectively ${ }^{13}$ (-Fig. $\mathbf{2}$ ).

\section{Clinical Guidelines}

One clinical guideline, produced by the American Association of Neurological Surgeons/Congress of Neurological Surgeons (AANS/CNS) Joint Section on Disorders of the Spine and Peripheral Nerves, was found that reviewed the evidence for the efficacy of bone growth stimulators as adjuncts for bone fusion following fusion surgery for degenerative disease of the lumbar spine. ${ }^{14}$ The authors concluded that there is insufficient evidence to recommend a treatment standard. These guidelines were based on evidence from four RCTs, three cohort studies, and two case series.

\section{Evidence Summary}

The overall strength of evidence evaluating the efficacy of electrical stimulation as an adjunctive treatment to promote bone fusion after lumbar spinal fusion procedures compared with no stimulation is low (-Table 2); that is, we have low confidence that evidence reflects the true effect and further research is likely to change the confidence in the estimate of effect and likely to change the estimate. With respect to DC stimulation, PEMF stimulation, and CC stimulation considered separately, the overall strength of evidence for each remains low.

\section{Discussion}

\section{- Strengths}

- The question was reviewed systematically.

\section{- Limitations}

- Few studies available to address the impact of different types of electrical stimulation.

- Heterogeneity among the individual studies precluded application of a meta-analysis.

- Random sequence generation, statement of concealed allocation, and intention-to-treat were reported very infrequently across the RCTs. 
Table 2 Evidence summary

\begin{tabular}{|l|l|l|l|}
\hline Outcomes & $\begin{array}{l}\text { Strength of } \\
\text { evidence }\end{array}$ & Conclusions/comments \\
\hline Compared with no stimulation, does electrical stimulation promote bone fusion after lumbar spinal fusion procedures? \\
\hline $\begin{array}{l}\text { Fusion: Any electri- } \\
\text { cal stimulation }\end{array}$
\end{tabular}

Abbreviations: CC, capacitive coupling; DC, direct current; PEMF, pulse electromagnetic field; RCT, randomized controlled trial.

- Loss to follow-up and controlling for possible confounding factors were not reported in two studies each, possibly biasing results.

- The use of a placebo device in the control group was not consistent across studies.

- Wide ranges of reported fusion rates (33.3 to 90.6\%) among the six RCTs are unexpected. Most notably, Anderson reported alarmingly low fusion rates of 33.3 and $35.4 \%$ in nonstimulated and stimulated groups at 24 months, respectively. This follow-up is longer than the other studies reviewed. However, it is important to note that their study was done in the elderly. Fusion is influenced by a multitude of factors such as age, sex, smoking status, surgical technique, grafts, and type of implants. In addition, the timing of fusion assessment and the criteria to determine fusion can vary. These factors likely explain the heterogeneity of outcomes reported.

- The implications in clinical practice of the use of electrical stimulation to promote bone fusion following lumbar spine surgery cannot be determined from the available evidence. However, it appears complications associated with its use are low.

- Additional large RCTs are warranted. Future RCTs need to focus on a single pathological process, encompass similar surgical procedures, and standardize electrical stimulation protocols. Clear a priori definitions of bone fusion need to be established and assessed by blinded reviewers.

\section{Acknowledgments}

Analytic support for this work was provided by Spectrum Research, Inc. with funding from AOSpine.

\section{Disclosures}

Paul Park, Consultant: Globus, Medtronic,

Biomet; Royalties: Globus

Darryl Lau, none

Erika D. Brodt, none

Joseph R. Dettori, none

\section{References}

1 Kornblum MB, Fischgrund JS, Herkowitz HN, Abraham DA, Berkower DL, Ditkoff JS. Degenerative lumbar spondylolisthesis with spinal stenosis: a prospective long-term study comparing fusion and pseudarthrosis. Spine (Phila Pa 1976) 2004;29(7):726-733, discussion 733-734

2 Nandyala SV, Marquez-Lara A, Fineberg SJ, Pelton M, Singh K. Prospective, randomized, controlled trial of silicate-substituted calcium phosphate versus rhBMP-2 in a minimally invasive transforaminal lumbar interbody fusion. Spine (Phila Pa 1976) 2014; 39(3):185-191

3 Martin BI, Mirza SK, Comstock BA, Gray DT, Kreuter W, Deyo RA. Reoperation rates following lumbar spine surgery and the influence of spinal fusion procedures. Spine (Phila Pa 1976) 2007; 32(3):382-387

4 Wright JG, Swiontkowski MF, Heckman JD. Introducing levels of evidence to the journal. J Bone Joint Surg Am 2003;85-A(1):1-3

5 Methods Guide for Effectiveness and Comparative Effectiveness Reviews. AHRQ Publication No. 10(12)-EHC063-EF. Rockville, MD: Agency for Healthcare Research and Quality; April 2012. Available at: www.effectivehealthcare.ahrq.gov

6 West S, King V, Carey TS, et al. Systems to Rate the Strength of Scientific Evidence. Evidence Report/Technology Assessment No. 47 (Prepared by the Research Triangle Institute-University of North Carolina Evidence-based Practice Center, Contract No. 290-97-0011). Rockville, MD: Agency for Healthcare Research and Quality; 2002 
7 Atkins D, Best D, Briss PA, et al; GRADE Working Group. Grading quality of evidence and strength of recommendations. BMJ 2004; 328(7454): 1490

8 Andersen T, Christensen FB, Egund N, et al. The effect of electrical stimulation on lumbar spinal fusion in older patients: a randomized, controlled, multi-center trial: part 2: fusion rates. Spine (Phila Pa 1976) 2009;34(21):2248-2253

9 Jenis LG, An HS, Stein R, Young B. Prospective comparison of the effect of direct current electrical stimulation and pulsed electromagnetic fields on instrumented posterolateral lumbar arthrodesis. J Spinal Disord 2000;13(4):290-296

10 Kane WJ. Direct current electrical bone growth stimulation for spinal fusion. Spine (Phila Pa 1976) 1988;13(3):363-365

11 Linovitz RJ, Pathria M, Bernhardt M, et al. Combined magnetic fields accelerate and increase spine fusion: a double-blind, ran-

\section{Editorial Perspective}

This systematic review regarding the efficacy of electrical stimulation for lumbar fusion surgery goes straight to the heart of one of the great unanswered questions of spinal reconstruction surgery: When has an arthrodesis surgery resulted in a "successful" fusion? After over six decades of performing fusion surgery, our spine community still remains less than clear on questions relating to the correlation of patient-related outcomes to fusion and radiographic confirmation of fusion. Efforts to predictably increase rates of fusion are aplenty and have left virtually no type of modulation affecting bone healing go unused.

Electric stimulation with its proven effect on bone healing had seemingly faded out of popularity after receiving a lot of attention in the 1990s. It is frustrating that despite seven high-grade, level-2 PRCTs in the end, the results of this systematic review remain ambiguous and do not provide meaningful guidance for practitioners interested in providing scientifically guided counseling to their patients. In reviewing domized, placebo controlled study. Spine (Phila Pa 1976) 2002; 27(13):1383-1389, discussion 1389

12 Mooney V. A randomized double-blind prospective study of the efficacy of pulsed electromagnetic fields for interbody lumbar fusions. Spine (Phila Pa 1976) 1990;15(7):708-712

13 Goodwin CB, Brighton CT, Guyer RD, Johnson JR, Light KI, Yuan HA. A double-blind study of capacitively coupled electrical stimulation as an adjunct to lumbar spinal fusions. Spine (Phila Pa 1976) 1999; 24(13):1349-1356, discussion 1357

14 Resnick DK, Choudhri TF, Dailey AT, et al; American Association of Neurological Surgeons/Congress of Neurological Surgeons. Guidelines for the performance of fusion procedures for degenerative disease of the lumbar spine. Part 17: bone growth stimulators and lumbar fusion. J Neurosurg Spine 2005;2(6):737-740

these studies in context with one another, the number of variables appears overwhelming.

Host factors (i.e., age, time from previous surgery, number of revisions, indication for index procedure, presence of complications, infection, diabetes, nicotine cessation), technique factors (quality of revision surgery, stability of construct stiffness, bone graft quality and quantity used, adjuvant bone growth factors employed, blood supply to host bone) all can play a significant role in bone healing. Establishing bone union itself remains unclear with computed tomography reformats, bone scans, dynamic radiographs, and surgical exploration with probing all remaining accepted forms of confirmation for fusion. Yet again, the resulting variables confound attempts at systematically evaluating the present day literature. The present study shows no conclusive evidence in support of using electric stimulation for routine lumbar fusion surgery. 